\title{
Overview on fosfomycin and its current and future clinical significance
}

\author{
Beata Chudzik-Rzad ${ }^{1 *}$, Sylwia Andrzejczuk ${ }^{1}$, Mariusz RzaD ${ }^{2}$, \\ Krzysztof TomasiewiCZ ${ }^{3}$, Anna Malm ${ }^{1}$
}

\begin{abstract}
${ }^{1}$ Department of Pharmaceutical Microbiology with Laboratory for Microbiological Diagnostics, Medical University of Lublin, Poland
${ }^{2}$ Public District Hospital in Krasnystaw, Poland

${ }^{3}$ Chair and Clinic Infection Diseases, Independent Public Teaching Hospital No 1 in Lublin, Poland
\end{abstract}

\begin{tabular}{|c|c|}
\hline ARTICLE INFO & ABSTRACT \\
\hline $\begin{array}{l}\text { Received } 02 \text { March } 2015 \\
\text { Accepted } 18 \text { March } 2015\end{array}$ & $\begin{array}{l}\text { Fosfomycin is an old antibiotic with a unique chemical structure and with broad- } \\
\text { spectrum activity against numerous bacterial pathogens, both Gram-positive and Gram- }\end{array}$ \\
\hline $\begin{array}{l}\text { Keywords: } \\
\text { fosfomycin, } \\
\text { urinary tract infections, } \\
\text { multi-resistant bacterial } \\
\text { infections. }\end{array}$ & $\begin{array}{l}\text { clinical practice in the early 1970s. Its use, however, has been limited for several years for } \\
\text { treating mainly lower uncomplicated urinary tract infections (in the form of fosfomycin } \\
\text { trometamol taken orally). Nowadays, many clinicians and scientists are looking at } \\
\text { this antibacterial drug for its employment in the treatment of severe infections caused } \\
\text { by multi-resistant bacteria. Fosfomycin as an intravenous formulation (fosfomycin } \\
\text { disodium) achieves clinically relevant concentrations in the serum and the cerebrospinal } \\
\text { fluid, in kidney, bladder wall, prostate, lungs, bone and heart valves tissues, as well as in } \\
\text { inflamed tissues and abscess fluid. The available clinical studies confirmed the efficacy } \\
\text { of intravenous fosfomycin for the management of severe infections caused by multi- } \\
\text { resistant pathogens. }\end{array}$ \\
\hline
\end{tabular}

\section{INTRODUCTION}

Fosfomycin, originally named 'phosphonomycin', was discovered in Spain, in 1969, being a product of native Streptomyces species, and its development for medical use began there in 1971. This antibiotic was initially used parenterally as the disodium salt form, for the treatment of serious infections, e.g. meningitis [4,33]. With time, its employment has been limited to treating lower uncomplicated urinary tract infections (in an oral formulation as fosfomycin trometamol) [29]. Now, in current medical practice, in the era of diminishing antibiotic options, a fosfomycin use renaissance is observable due to its good activity against resistant and multi-resistant bacteria [7,22,29,32].

\section{FOSFOMYCIN - GENERAL INFORMATION}

Fosfomycin ([(2R,3S)-3-methyloxiran-2-yl]phosphonic acid), of the family of phosphonic antibiotics, is an unique antibiotic of a chemical structure unrelated to those of any other known antibacterial agent $[4,21,29,33]$. Fosfomycin

\footnotetext{
* Corresponding author

e-mail: beata.chudzik-rząd@umlub.pl
}

is a bactericidal agent that inhibits bacterial cell wall biosynthesis by acting on the initial stages in the synthesis of peptidoglycan procedures: the inhibition of UDP-N-acetylglucosamine enolpyruvyl transferase (MurA) [1,4,10,21,33]. This enzyme is responsible for the synthesis of UDP-Nacetylenolpyruvylglucosamine, which is a pentapeptide intermediate in the biosynthesis of the peptidoglycan layer of the bacterial cell wall [28]. Due to its different chemical structure and mechanism of action, fosfomycin does not have cross resistance with the currently used drugs [29].

Fosfomycin has a broad spectrum of antimicrobial activity, including that upon several Gram-negative and Gram-positive aerobic pathogens [14,33]. The activity of fosfomycin depends on its transport into bacterial cell by way of transport systems for L- $\alpha$-glycerophosphate and hexose monophosphate, involving the presence of glucose6-phosphate. Because of this reason, the determination of antimicrobial activity of fosfomycin in vitro requires an addition to the medium of glucose-6-phosphate [33].

Fosfomycin has a serum half-life of approximately 1,75 hours [1]. However, it shows post-antibiotic effects in vitro against E. coli or P. mirabilis, varying between $3.4 \mathrm{~h}$ and 
$4.7 \mathrm{~h}$, depending on the applied drug concentration [28]. Fosfomycin also exerts time-dependent bactericidal activity against both Gram-negative and Gram-positive pathogens [29]. The antibiotic is available as intravenous formulation (fosfomycin disodium) and as oral formulations (fosfomycin trometamol - a salt of fosfomycin with tromethamine - and fosfomycin calcium) $[4,29,33]$.

\section{FOSFOMYCIN AND URINARY TRACT INFECTIONS}

Fosfomycin is currently used mostly as fosfomycin trometamol and is taken orally. This formulation is approved in numerous countries for treating lower uncomplicated urinary tract infections, e.g. acute cystitis, due to its good activity against common uropathogens, especially Escherichia coli and Enterococcus faecalis, as well as its good tolerability and safety. Furthermore, it reaches very high and persistent urinary concentrations (up to $48 \mathrm{~h}$ ) $[2,12,38]$. A single oral dose of fosfomycin trometamol (the approved dosage) is $3 \mathrm{~g}$. This antibiotic is also recommended for prophylaxis of recurrent urinary tract infections or in urological diagnostic procedures and surgery [29].

Results of the clinical trials indicate that single-dose fosfomycin trometamol has similar clinical and/or bacteriological effects comparable to 3-7-day regimens of ciprofloxacin, norfloxacin, cotrimoxazole or nitrofurantoin in women with uncomplicated lower urinary tract infections [23]. In addition, single-dose fosfomycin trometamol has similar bacteriological efficacy to a 5-day course of cefuroxime axetil or a 7-day course of amoxicillin/clavulanic acid in pregnant women with asymptomatic bacteriuria, and similar clinical and/or bacteriological efficacy to a 5-day course of cefuroxime axetil or amoxicillin/clavulanic acid in pregnant women with lower urinary tract infections [23]. It should be noted that fosfomycin trometamol is recommended for the treatment of urinary tract infections during pregnancy, and belongs to the Food and Drug Administration category B [5]. Moreover, Ruxer et al. [35] have confirmed the efficacy of chronic therapy with fosfomycin, co-trimoxazole and nitrofurantoin in the treatment and prevention of recurrent urinary tract infections in type 2 diabetic women, and drew a conclusion that the prophylactic use of fosfomycin was rarely associated with recurrence of these infections.

Lu et al. [27] studied the activity of fosfomycin against 960 strains of common uropathogens, including 3 common species of Enterobacteriaceae (Escherichia coli, Klebsiella pneumoniae, Enterobacter cloacae), non-fermentative rods - Pseudomonas aeruginosa, Acinetobacter baumannii, and Stenotrophomonas maltophilia, methicillin-susceptible and -resistant Staphylococcus aureus, vancomycin-susceptible and -resistant Enterococcus faecalis and Enterococcus faecium. It was found that $E$. coli, the most common pathogen of urinary tract infections, was invariably susceptible to fosfomycin, while the prevalence of fosfomycinresistance among other pathogens was variable. In addition, Sultan et al. [36], performing studies on a total of 1840 urine samples, found that fosfomycin was $100 \%$ effective against methicillin-resistant $S$. aureus (MRSA), vancomycin-resistant enterococci (VRE), enterococci with high-level aminoglycoside resistance (HLAR) and Gram-negative bacteria producing extended-spectrum beta-lactamases (ESBL). Besides the aforementioned, $99 \%$ of Gram-negative bacteria producing beta-lactamases AmpC were found to be sensitive to fosfomycin. The above data indicate that fosfomycin may still be regarded as a promising option for treatment of urinary tract infections, especially in that it is useful in cases involving multi-resistant pathogens that are resistant to currently used treatments of urinary tract infections.

Although prostatic infections due to multidrug-resistant Enterobacteriaceae are an increasing clinical problem, Gardiner et al. [16] found that oral fosfomycin achieves sufficient intraprostatic concentrations in healthy men to be potentially effective in preventing prostatic infection in patients undergoing transrectal ultrasound-guided biopsy, and to be considered as a therapeutic option for patients with prostatitis caused by multidrug-resistant Enterobacteriaceae. However, further clinical studies are required.

\section{FOSFOMYCIN BEYOND URINARY TRACT INFECTIONS}

Nowadays, many clinicians and scientists look at fosfomycin as alternative therapeutic option in infectious diseases caused by multi-resistant pathogens [7,22,29,32]. These pathogens were recently deemed ESCAPE pathogens (Enterococcus faecium, Staphylococcus aureus, Klebsiella pneumoniae, Acinetobacter baumannii, Pseudomonas aeruginosa, Enterobacter spp.) due to their "escape" from available antimicrobial agents, as well as the non-existence of newer active antibiotics [6].

Fosfomycin has not been widely used as other drugs. Indeed, the intravenous formulation has been employed for indications beyond uncomplicated urinary tract infections in only a few countries (such as Germany, France, Spain or Japan) [12]. In some European countries, however, fosfomycin has been occasionally administered for the initial empirical therapy of sepsis or soft-tissue infections. Resistance to fosfomycin is not widespread due to its limited usage, and the emergence of resistance to fosfomycin has been found to only occur in 2.3-6.7\% of all cases in which this agent has been used for the treatment of infections other than uncomplicated cystitis, such as respiratory tract infections or osteomyelitis [22]. It should be noted that several mechanisms of resistance to fosfomycin have been described, including decreased drug uptake, modification of the target site and inactivation of the antibiotic [23].

Several studies have been performed on the in vitro activity of fosfomycin against resistant or multi-resistant bacteria. Kasse et al. [20], in testing susceptibility to fosfomycin in a collection of 107 carbapenem-nonsusceptible clinical isolates belonging to Enterobacteriaceae (of which 80 produced various types of carbapenemases), found that, overall, $78 \%$ of the strains were sensitive to fosfomycin. Beyond this work, Livermore et al. [26] determined the activity of some antimicrobial agents against Enterobacteriaceae with resistance to carbapenems, including fosfomycin. They found that fosfomycin was active against $49 / 81(60.5 \%)$ of the isolates belonging to carbapenemresistant Enterobacteriaceae, including 7/7 Escherichia coli, 16/20 Enterobacter spp. and Citrobacter spp., but only 
was effective against 25/52 of Klebsiella spp. As reported by Fagalas et al. [14], fosfomycin had good antibacterial activity against the clinical isolates of Enterobacteriaceae that produced ESBL, being useful against $90 \%$ or more of them. Interestingly, high activity of fosfomycin has been reported against isolates of Enterobacteriaceae with resistance to fluoroquinolones [24]. Of note, E. coli seems to be the most susceptible to fosfomycin among Enterobacteriaceae, regardless of the isolate origin, i.e. clinical specimens in community- and hospital-acquired infections [14].

Fosfomycin was found to possess promising activity against carbapenemase-producing $P$. aeruginosa and $K$. pneumoniae, and to other multi-resistant strains such as meticilin-resistant $S$. aureus (MRSA) and extensively drug resistant $P$. aeruginosa [15,30,31]. Fagalas et al. [13] collected data published in website databases, regarding the clinical and/or microbiological effectiveness of fosfomycin in the treatment of infections caused by multi-resistant, extensively drug-resistant or pandrug-resistant non-fermenting Gram-negative bacilli. From this report comes one conclusion - fosfomycin can have an important role as a therapeutic option against $P$. aeruginosa infections. Moreover, the susceptibility to fosfomycin of multi-resistant $P$. aeruginosa isolates varied between $50 \%$ and $\geq 90 \%$, depending on the study. That which was most susceptible to fosfomycin were isolates of $P$. aeruginosa of serotype O12. On the other hand, only $3.5 \%$ of all multi-resistant A. baumannii isolates and no isolates of Burkholderia spp. were susceptible to fosfomycin.

Allberger and Ingo [1] studied sensitivity to fosfomycin among 69 vancomycin-resistant isolates of Enterococcus spp., including: E. faecium and E. faecalis with phenotype Van A and Van B, E. gallinarum with phenotype Van C1 and E. casseliflavus with phenotype Van C2. They observed a high rate of fosfomycin-mediated inhibition of the examined enterococci, this figure being $94-97 \%$, and concluded that fosfomycin, when used in combination with other drugs, could be a potentially useful drug for the treatment of infections caused by vancomycin-resistant enterococci. It should be realised that new therapeutic strategies are needed to combat the emergence of infections due to multidrugresistant Neisseria gonorrhoeae. Fosfomycin was found to be active in vitro against the gonococci isolates possessing resistance to ceftriaxone and/or azithromycin [19].

Fosfomycin is effective against a wide range of infections caused by Gram-negative and Gram-positive bacteria, especially in patients suffering from multi-resistant bacterial infections. However, clinical studies to assess the efficacy of fosfomycin are limited [34]. Based on the fact that fosfomycin monotherapy with intravenous formulation should be prohibited due to the prompt emergence of resistance during therapy, the choice of the appropriate adjunctive antibiotic should be carefully investigated and must be individualized for each patient's condition $[9,17]$. In a prospective study, fosfomycin was given at 2-4 g intravenously every $6 \mathrm{~h}$ for $14 \pm 5.6$ days, in combination with other antibiotics, to 11 adults hospitalized in an Intensive Care Unit due to carbapenem-resistant $K$. pneumoniae infections without definition of underlying resistance mechanisms [30]. All patients had promising bacteriological and clinical outcomes, with all-cause hospital mortality of $18 . \%$. Additionally, no adverse effects were reported. Chen et al. [9] suggest that combination therapy consisting of high-dose daptomycin and fosfomycin is an alternative treatment option for daptomycin-nonsusceptible, methicilin-resistant $S$. aureus native valve endocarditis or osteomyelitis. Especially, in that these two antibacterial agents are well known for their bacteriocidal activities and effective biofilm penetration $[3,11]$. Of note, infective endocarditis is a clinically significant disease with mortality ranges from $16 \%$ to $25 \%$ [11].

Beyond the aforementioned, Hamilton-Miller [18] found that fosfomycin could be a useful addition to the limited number of antimicrobial drugs, available in clinical practice effective against multi-resistant Gram-positive cocci. Fosfomycin may, thus, be regarded as a valuable option in combination therapy in the treatment of infections due to resistant and multi-resistant pathogens in haematology patients and haematopoietic stem cell transplant recipients [37]. Additionally, recent study implies that fosfomycin can be an alternative to the current ampicillin treatment of listeriosis in humans [25].

Moreover, fosfomycin is also considered as an antibacterial agent useful for perioperative prophylaxis. Chareancholvanich et al. [8] studying the safety and efficacy of fosfomycin and cefuroxime as antibiotic prophylaxis for knee arthroplasty, suggest using fosfomycin. They put forward that it is a safe and effective antibacterial agent - no patients had any complication and none had renal and liver function disturbance during the follow-up.

It should be noted that fosfomycin as intravenous formulation (fosfomycin disodium) possesses good pharmacokinetic properties, including its distribution into tissues. This antibiotic achieves clinically relevant concentrations in serum, kidneys, bladder wall, prostate, lungs, bone, cerebrospinal fluid, and heart valves. Beyond this, therapeutic concentrations were found to be within inflamed tissues and abscess fluid [29].

\section{CONCLUSION}

Fosfomycin is an old antibiotic and its effectiveness may be to some extent, underestimated. Nowadays, however, many clinicians and scientists are having a re-look at this antibacterial drug in the light of its good activity against multi-resistant microorganisms - these being etiological factors of severe infections both in hospitalized and ambulatory patients.

\section{REFERENCES}

1. Allerberger F., Klare I.: In-vitro activity of fosfomycin against vancomycin-resistant enterococci. J. Antimicrob. Chemother., 43, 211, 1999.

2. Bader M.S., Hawboldt J., Brooks A.: Management of complicated urinary tract infections in the era of antimicrobial resistance. Postgrad. Med., 122, 7, 2010.

3. Baltz R.H., Daptomycin: mechanisms of action and resistance and biosynthetic engineering. Curr. Opin. Chem. Biol., 13, 144, 2009.

4. Baylan O.: Fosfomycin: past, present and future. Mikrobiyol. Bul., $44,311,2010$. 
5. Bayrak Ö. et al.: Is single-dose fosfomycin trometamol a good alternative for asymptomatic bacteriuria in the second trimester of pregnancy? Int. Urogynecol. J., 18, 525, 2007.

6. Bocher H.W. et. al.: Bad bugs, no drugs: no ESCAPE! An update from the Infectious Diseases Society of America. Clin. Infect. Dis., $48,1,2009$.

7. Cassir N., Rolain J.M., Brouqui P.: A new strategy to fight antimicrobial resistance: the revival of old antibiotics. Front. Microbiol., 5, 1, 2014.

8. Chareancholvanich K., Udomkiat P., Waikakul S.: A randomized control trial between fosfomycin and cefuroxime as the antibiotic prophylaxix in knee arthroplasty. J. Med. Assoc. Thai., 9, 6, 2012.

9. Chen L.Y. et al.: High-dose daptomycin and fosfomycin treatment of a patient with endocarditis caused by daptomycin-nonsusceptible Staphylococcus aureus: Case report. BMC. Infect. Dis., 11, 1, 2011.

10. Eschenburg S., Priestman M., Schonbrunn E.: Evidence that the fosfomycin target Cys115 in UDP-N-acetylglucosamine enolpyruvyl transferase (MurA) is essential for product release. J. Biol. Chem., 280, 3757, 2005.

11. Falagas M.E. et al.: Daptomycin for endocarditis and/or bacteraemia: a systematic review of the experimentaland clinical evidence. $J$. Antimicrob. Chemother., 60, 7, 2007.

12. Falagas M.E. et al.: Fosfomycin: use beyond urinary tract and gastrointestinal infections. Clin. Infect. Dis., 46, 1069, 2008.

13. Falagas M.E. et al.: Fosfomycin for the treatment of infections caused by multidrug-resistant non-fermenting Gram-negative bacilli: a systematic review of microbiological, animal and clinical studies. Int. J. Antimicrob. Agents., 34, 111, 2009.

14. Falagas M.E.et al.: Fosfomycin for the treatment of multidrugresistant, including extended-spectrum $\beta$-lactamase producing, Enterobacteriaceae infections: a systematic review. Lancet. Infect. Dis., 10, 43, 2010.

15. Falagas M.E. et al.: Antimicrobial susceptibility of multidrugresistant (MDR) and extensively drug-resistant (XDR) Enterobacteriaceae isolates to fosfomycin. Int. J. Antimicrob. Agents., 35, 240, 2010.

16. Gardiner B.J., et al.: Is fosfomycin a potential treatment alternative for multidrug-resistant gram-negative prostatitis? Clin. Infect. Dis., $58,101,2014$.

17. Giamarellou H.: Multidrug-resistant Gram-negative bacteria: how to treat and for how long. Int. J. Antimicrob. Agents., 36, 50, 2010.

18. Hamilton-Miller J.M.: In vitro activity of fosfomycin against "problem" Gram-positive cocci. Microbios., 1992, 71: 95-103.

19. Hauser C. et al.: In vitro activity of fosfomycin alone and in combination with ceftriaxone or azithromycin against clinical Neisseria gonorrhoeae isolates. Antimicrob. Agents. Chemother., 59, $1605,2015$.

20. Kaase M. et al.: Fosfomycin in susceptibility in carbapenem-resistant Enterobacteriaceae from Germany. J. Clin. Microbiol. 52, 1893, 2014

21. Kahan FM. et al.: The mechanism of action of fosfomycin (phosphonomycin). Ann. N. Y. Acad. Sci., 235, 364, 1974.
22. Karageorgopoulos D.E. et al.: Fosfomycin: evaluation of the published evidence on the emergence of antimicrobial resistance in Gram-negative pathogens. J. Antimicrob. Chemother., 67, 255, 2012.

23. Keating G.M.: Fosfomycin trometamol: a review of its use as a single-dose oral treatment for patients with acute lower urinary tract infections and pregnant women with asymptomatic bacteriuria. Drugs., 73, 1951, 2013.

24. Ko K.S. et al.: In vitro activity of fosfomycin against ciprofloxacinresistant or extended-spectrum $\beta$-lactamase-producing Escherichia coli isolated from urine and blood. Diagn. Microbiol. Infect. Dis., $58,111,2007$.

25. Lepe J.A. et al.: In vitro and intracellular activities of fosfomycin against clinical strains of Listeria monocytogenes. Int. J. Antimicrob. Agents., 43, 135, 2014.

26. Livermore D.M. et al.: What remains against carbapenem-resistant Enterobacteriaceae? Evaluation of chloramphenicol, ciprofloxacin, colistin, fosfomycin, minocycline, nitrofurantoin, temocyllin and tigecycline. Int. J. Antimicrob. Agents., 37, 415, 2011.

27. Lu C.L. et al.: Antimicrobial susceptibilities of commonly encountered bacterial isolates to fosfomycin determined by agar dilution and disk diffusion methods. Antimicrob. Agents Chemother., $55,4295,2011$.

28. Mazzei T. et al.: Pharmacokinetic and pharmacodynamic aspects of antimicrobial agents for the treatment of uncomplicated urinary tract infections. J. Antimicrob. Agents., 28, 35, 2006.

29. Michalopoulos A.S., Livaditis I.G., Gougoutas V.: The revival of fosfomycin. Int. J. Infect. Dis., 15, 732, 2011.

30. Michalopoulos A. et al.: Intravenous fosfomycin for the treatment of nosocomial infections caused by carbapenem-resistant Klebsiella pneumoniae in critically ill patients: a prospective evaluation. Clin. Microbiol. Infect., 16, 184, 2010.

31. Pontikis K. et al.: Outcomes of critically ill intensive care unit patients treated with fosfomycin for infections due to pandrugresistant and extensively drug-resistant carbapenemase-producing Gram-negative bacteria. Int. J. Antimicrob. Agents., 43, 52, 2014.

32. Poulakou G. et al.: Current and future treatment options for infections caused by multidrug-resistant Gram-negative pathogens. Future. Microbiol., 9, 1053, 2014.

33. Raz R.: Fosfomycin: an old-new antibiotic. Clin. Microbiol. Infect., $18,4,2012$.

34. Reffert J.L., Smith W.J.: Fosfomycin for the treatment of resistant gram-negative bacterial infections. Insights from the society of infectious diseases pharmacists. Pharmacotherapy., 34, 845, 2014.

35. Ruxer J. et al.: Fosfomycin, co-trimoxazole and nitrofurantoin in the treatment of recurrent uncomplicated urinary tract infections in type 2 diabetes mellitus. Wiad. Lek., 60, 235, 2007.

36. Sultan A. et al.: Increasing antimicrobial resistance among uropathogens: is fosfomycin the answer? Urol. Ann., 7, 26, 2015.

37. Trubiano J.A. et al.: The prevention and management of infections due to multidrug resistant organisms in haematology patients. $B r$. J. Clin. Pharmacol., 79, 195, 2015.

38. Wang A. et al.: Urinary tract infections. Prim. Care., 40, 687, 2013. 